\title{
Acute Stroke with Hyperdense Middle Cerebral Artery Sign Benefits from IV rtPA
}

\author{
Tartaglia MC, Di Legge S, Saposnik G, Jain V, Chan R, Bussière M, Hachinski V, \\ Frank $C$, Hesser $K$, Pelz D
}

\begin{abstract}
Objectives: The current management of acute ischemic stroke is intravenous (IV) recombinant tissue plasminogen activator (rtPA). The presence of a hyperdense middle cerebral artery sign (HMCAS) on pre-treatment head computed tomogram (CT) is considered a poor prognostic sign. We compared the clinical outcome in IV rtPA-treated patients with and without a HMCAS. Design: Retrospective analysis of prospectively collected cases treated with IV rtPA within three hours. Inclusion criteria were the presence of: i) an anterior circulation stroke; ii) a pre-treatment CT available; iii) a pre-treatment National Institutes of Health (NIH) stroke scale (NIHSS) score; and iv) a modified Rankin Score (mRS) at three months. Results: One hundred and thirty patients were eligible for the analysis, $64(49 \%)$ had a HMCAS. The HMCAS group had a trend toward a higher mean $( \pm \mathrm{SD})$ pre-treatment NIHSS score compared to the non-HMCAS group ( $13.9 \pm 6$ vs. $12.2 \pm 6 ; \mathrm{p}=0.12)$. Accordingly, there were more patients with severe strokes (NIHSS $>10)$ in the HMCAS group compared to the non-HMCAS one $(48 / 64=75 \%$ vs. $35 / 66=53 \% ; p=0.009)$. The mean $( \pm$ SD) NIHSS score 24 hours after treatment was $10.6( \pm 8)$ in the HMCAS group and $8.3( \pm 7)$ in the non-HMCAS group $(\mathrm{p}=0.08)$. In a multiple logistic regression analysis, the only independent predictor of poor outcome (mRS 3-6) was pre-treatment NIHSS score ( $<0.001)$. Conclusion: Patients with a HMCAS receiving IV rtPA did not fare worse at three months despite a greater proportion of patients with more severe strokes. Based on the current knowledge, IV rtPA remains a good treatment for patients with a HMCAS within three hours of symptom onset.
\end{abstract}

RÉSUMÉ: L'accident vasculaire cérébral aigu avec signe de l'artère cérébrale moyenne hyperdense bénéficie de l'administration de rt-PA IV. Objectifs : L'administration de l'activateur du plasminogène tissulaire recombinant (rt-PA) est le traitement actuel de l'accident vasculaire cérébral $(\mathrm{AVC})$ ischémique aigu. La présence du signe de l'artère cérébrale moyenne hyperdense (SACMH) à la tomodensitométrie cérébrale avant traitement est considérée comme un signe de mauvais pronostic. Nous avons comparé le résultat clinique chez les patients avec et sans SACMH traités par rt-PA IV. Plan de l'étude : Nous avons effectué une analyse rétrospective de cas recueillis de façon prospective, traités au moyen de rt-PA IV dans les 3 heures du début des symptômes. Les critères d'inclusion étaient les suivants : la présence d'un AVC dans le territoire de la circulation antérieure ; la disponibilité d'un examen tomodensitométrique avant traitement, d'un score du NIH Stroke Scale (NIHSS) avant traitement et d'un score du Modified Rankin Scale (MRS) trois mois après l'AVC. Résultats : Cent trente patients rencontraient les critères d'inclusion, dont soixante-quatre (49\%) avaient un SACMH. Dans le groupe avec SACMH, la moyenne du score du NIHSS avant traitement avait tendance à être plus élevée comparée à celle du groupe sans SACMH $(13,9 \pm 6$ vs $12,2 \pm 6 ; \mathrm{p}=0,12)$. Il y avait donc plus de patients atteints d'AVC sévères (NIHSS $>10)$ dans le groupe avec SACMH par rapport au groupe sans SACMH $(48 / 64=75 \%$ vs $35 / 66=53 \% ; p=0,009)$. Le score moyen du NIHSS 24 heures après le traitement était de 10,6 ( \pm 8) dans le groupe avec SACMH et de 8,3 $( \pm 7)$ dans le groupe sans SACMH $(\mathrm{p}=0,08)$. À l'analyse de régression logistique multivariée, le seul prédicteur indépendant d'un mauvais pronostic (MRS 3-6) était le score du NIHSS avant traitement ( $<<0,001)$. Conclusion : Les patients qui présentaient un SACMH et qui ont reçu du rt-PA IV n'étaient pas en plus mauvais état trois mois plus tard que ceux qui n'en présentaient pas malgré qu'une plus grande proportion d'entre eux étaient atteints d'AVC plus sévères. Selon les connaissances actuelles, le rt-PA IV administré dans les trois heures du début des symptômes est un bon traitement chez les patients porteurs d'un SACMH.

Can. J. Neurol. Sci. 2008; 35: 583-587

Intravenous (IV) delivery of recombinant tissue plasminogen activator (rtPA) within three hours of symptom onset is the first treatment option for acute ischemic stroke ${ }^{1}$. A hyperdense middle cerebral artery (HMCAS) sign on baseline CT scan correlates with proximal middle cerebral artery (MCA) occlusion on CT angiography ${ }^{2}$, is associated with higher baseline National Institutes of Health Stroke Scale (NIHSS) scores ${ }^{3}$, larger cerebral infarction on follow-up imaging ${ }^{4-9}$, and worse outcome at three months ${ }^{3}$.
From the Department of Clinical Neurological Sciences (MCT, RC, MB, VH, CF, KH), Department of Diagnostic Radiology and Nuclear Medicine (DP), London Health Sciences Center, University of Western Ontario, London, ON; Stroke Unit, University of Tor Vergata (SDL), Viale Oxford 81-00133 Rome, Italy; Department of Medicine, St. Michael's Hospital (GS), University of Toronto, Ontario, Canada; Division of Research (VJ), Kaiser-Permanente Northern California, Oakland, CA, USA. Received April 24, 2008. Final Revisions Submitted June 4, 2008. Correspondence to: Carmela Tartaglia, Division of Neurology, Dept. Clinical Neurological Sciences, University of Western Ontario, B7-005, 339 Windermere Rd, London, Ontario, N6A 5A5, Canada. 
A less favourable outcome was reported in patients with a HMCAS receiving IV rtPA compared to patients without a HMCAS. ${ }^{3,10,11}$ However, in the European cooperative acute stroke study (ECASS) cohort, patients with a HMCAS did better if they received IV rtPA compared to placebo, and the presence of a HMCAS on pre-treatment CT scan was not an independent predictor of poor outcome. ${ }^{12}$ A very recent, retrospective study reported a better outcome in HMCAS patients who had received IA rtPA within six hours compared to HMCAS patients who had received IV rtPA within three hours. There were, however, differences between the two groups in that many more IA rtPAtreated patients had cardioembolic strokes and many more IV rtPA-treated patients had strokes due to large artery disease. It is unknown what effect this may have had on the results. It has been reported that the less favourable outcome observed in patients with HMCAS may be due to more severe strokes at baseline, but they were nonetheless, more likely to attain a favourable outcome after IV rtPA. ${ }^{13}$

It is not clear, however, whether a HMCAS (i.e. the presence of proximal MCA occlusion) warrants more aggressive intraarterial thrombolysis or combined IV + IA thrombolysis. ${ }^{14,15}$ The rate of early recanalization of MCA stems or divisions by conventional angiography was $38 \%$ in IV rtPA patients with pretreatment HMCAS. ${ }^{16}$ A number of studies have shown that IA alone or in combination with IV delivery of thrombolytics increased the chance of recanalization either compared to placebo ${ }^{17}$ or to the IV route alone. ${ }^{18}$ Combination of IV and IA routes and using solely the IA route within six hours improved outcome for the patients suffering from occlusion of the distal internal carotid artery. ${ }^{19}$

In many institutions, acute stroke patients who reach the hospital beyond the three-hour window can be offered, in selected cases, the possibility to receive IA rtPA or mechanical therapies. A recent retrospective analysis showed that patients with a HMCAS had a better outcome at three-months if treated with IA rtPA despite treatment being delayed beyond the threehour window as compared to the IV route. ${ }^{20}$ The dilemma, however, remains for those patients with evidence of HMCAS who arrive within three hours: should these patients receive IV rtPA (i.e. the standard treatment) with no further delay, or should they be offered IA rtPA if further investigations detect major artery occlusion and viable tissue? A randomized double-blind trial of IA thrombolysis vs. IV thrombolysis within six hours of symptom onset is ongoing. ${ }^{21}$

Overall there is little prospective data available on the correlation between pre-treatment CT status and long-term outcome after thrombolysis, and a retrospective study may still be useful to address these issues until prospective data are available. This, in turn, might lead to the development of predefined algorithms that might allow the medical team to offer the best available treatment specific to each patient.

We hypothesized that stroke patients with a MCA occlusion as suggested by the presence of a HMCAS on pre-treatment head CT scan have poorer outcome than patients without major arterial steno-occlusive disease and consequently may potentially benefit from more aggressive intervention (i.e. IA or IV+IA rtPA). For this purpose we determined if the presence of a HMCAS on pre-treatment CT scan was a predictor of poor outcome at three months in patients treated with IV rtPA.

\section{METHODS}

This is a retrospective analysis of patients selected from a registry of acute ischemic stroke patients who had received thrombolysis (IV, IA or combined treatment) between December 1998 and March 2005. We included all IV rtPA-treated patients with the following inclusion criteria: i) had received IV rtPA within the three-hour window; ii) had suffered from an anterior circulation stroke in the MCA territory; iii) had pre-treatment CT scan available; iv) had pre-treatment NIHSS score available; v) had a three-month follow-up modified Rankin Score (mRS) recorded. We excluded patients with posterior circulation strokes and patients who had received IA rtPA alone or in combination with IV treatment. Demographic, clinical and outcome data were prospectively collected and entered into a database, details of which have been reported elsewhere..$^{22-24}$ Non-contrast CT scans of the head performed before and after thrombolysis were retrospectively reviewed for evidence of a HMCAS and "dot" signs by two trained stroke neurologists (MCT and SDL) blinded for the side of the affected body and for the site of infarction on follow-up CT scan. The HMCAS was defined as a MCA denser than its counterpart and denser than any other visualized artery or vein. The HMCAS was categorized according to its location as "proximal" (MCA trunk or basal M1 segment), "distal" (beyond MCA bifurcation or M2 and M3 segments) i.e. the "dot sign", or both. The HMCAS was interpreted as "present" or "absent," and doubtful cases were considered to be absent. In case of disagreement the two came to a final judgment during a consensus meeting.

Stroke severity was assessed based on the NIH Stroke Scale. ${ }^{25}$ A severe stroke was defined as an NIHSS $>10$ given that a previous study revealed that $46 \%$ of patients with onset NIHSS 7-10 had a good recovery and this dropped dramatically to $23 \%$ in the NIHSS 11-15 group. ${ }^{26}$ Functional outcome at three months was assessed with the mRS ( 0 to 6 , where 6 defines death). ${ }^{27}$

Patients with the hyperdense MCA sign (HMCAS group) were compared with those who did not have the sign (nonHMCAS group) on baseline CT scan in terms of: i) demographics; ii) stroke risk factors ( i.e. arterial hypertension, smoking, diabetes mellitus, hypercholesterolemia, previous transient ischemic attack (TIA), previous stroke, coronary artery disease, atrial fibrillation, peripheral vascular disease and obesity); iii) pre-treatment NIHSS; iv) onset-to-needle time (minutes); v) NIHSS at 24 hours after symptom onset; vi) length of hospital stay (days); vii) occurrence of symptomatic haemorrhage; and viii) three-month outcome as assessed by the mRS (good outcome 0-2, poor outcome 3-6).

Statistical analysis: Comparison of patients with and without HMCAS was performed with t-test, Mann-Whitney or chisquare test, as appropriate. For continuous data, we used student's t test and ANOVA. A multiple logistic regression was performed with Modified Rankin Score as the dependent variable dichotomized for good outcome 0-2 and poor outcome 3-6 and the following independent variables: age, gender, pretreatment NIHSS, presence or absence of a HMCAS, presence or absence of the following risk factors: hypertension, smoking status, diabetes mellitus, hypercholesterolemia, previous TIA, previous stroke, coronary artery disease, atrial fibrillation, peripheral vascular disease and obesity. Significance was set at 


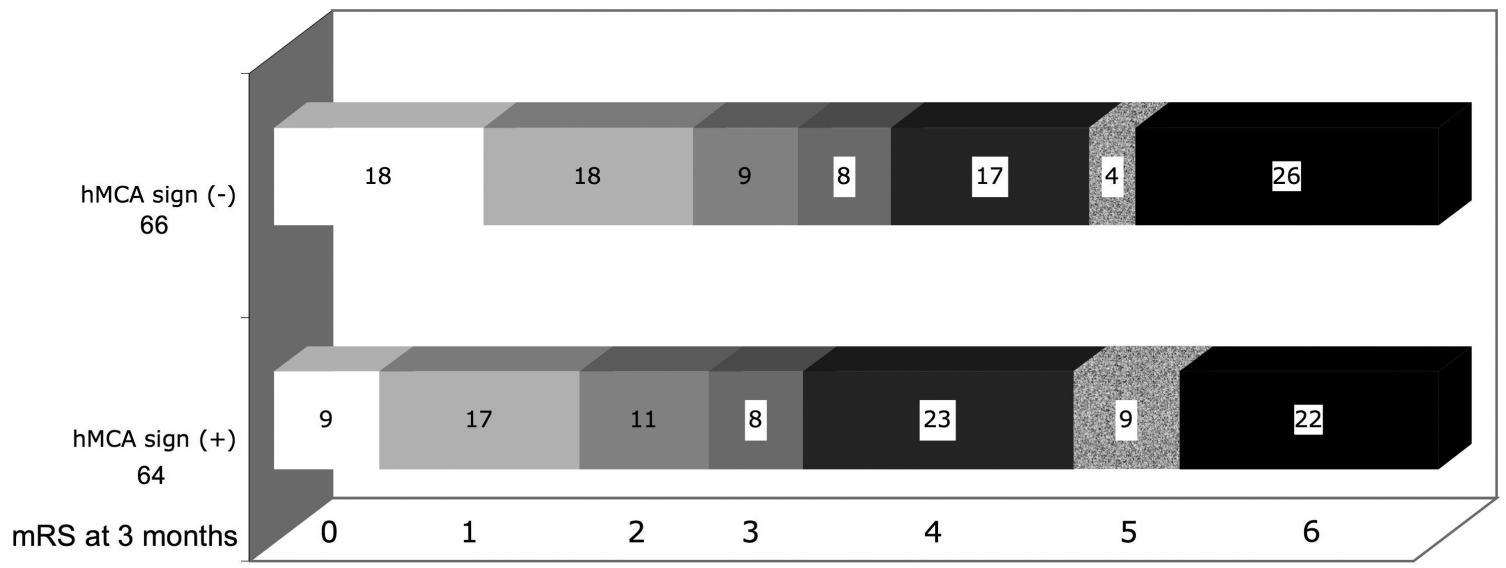

Figure: Percentage of patients with and without hyperdense MCA at each Modified Rankin score at three months.

$\mathrm{P}<0$.05. All tests were 2-sided and computed with the software SPSS 11.

\section{Results}

There were 312 patients who had received IV and/or IA rtPA for an acute ischemic stroke in our institution between December 1998 and March 2005. Of them, 130 (42\%) patients met the inclusion criteria (i.e., anterior circulation infarct in the MCA territory, pre-treatment CT scan available, pre-treatment NIHSS score, and three-month mRS). Of the remaining 182 patients, 10 had received either IA rtPA alone or a combination of IV and IA rtPA treatment, 30 had a posterior circulation stroke, and 142 had incomplete data. Hence, for the purpose of this study we ran the

Table 1: Prevalence of risk factors in patients with and without HMCAS

\begin{tabular}{lccc}
\hline & HMCAS(+) & HMCAS(-) & P value \\
& $\mathbf{6 4}(\boldsymbol{\%})$ & $\mathbf{6 6}(\boldsymbol{\%})$ & \\
hypertension & $42(65)$ & $38(58)$ & 0.37 \\
smoking & $13(20)$ & $12(18)$ & 0.83 \\
diabetes mellitus & $13(20)$ & $16(24)$ & 0.68 \\
hypercholesterolemia & $28(44)$ & $22(33)$ & 0.28 \\
previous TIA & $9(14)$ & $8(12)$ & 0.8 \\
previous stroke & $17(27)$ & $9(14)$ & 0.08 \\
coronary artery disease & $20(31)$ & $21(32)$ & 1 \\
atrial fibrillation & $23(36)$ & $17(26)$ & 0.26 \\
peripheral vascular disease & $3(5)$ & $4(6)$ & 1 \\
obesity & $7(11)$ & $5(8)$ & 0.56 \\
\hline
\end{tabular}

analyses on 130 patients. Of these, 64/130 (49\%) had a HMCAS or "dot" sign (hereafter called HMCAS group) and 66/130 (51\%) did not (hereafter called the non-HMCAS group). There was a significantly higher prevalence of males in the HMCAS group compared to the non-HMCAS group ( $66 \%$ vs. $38 \%$; $\mathrm{p}=0.001)$, while mean age $( \pm \mathrm{SD})$ did not differ between the two groups (73.3 \pm 12 vs. $70.4 \pm 13)$. There was a significantly lower percentage of right hemispheric strokes in the HMCAS group ( $28 \%$ vs. $45 \% ; \mathrm{p}=0.001)$. The two groups did not differ significantly for vascular risk factors except for a trend towards a higher occurrence in the HMCAS group of previous ischemic stroke (17\% vs. $9 \%$; $\mathrm{p}=0.08)$ (Table 1$)$. Mean $( \pm \mathrm{SD})$ onset-totreatment time was similar between the two groups: $156( \pm 36)$ minutes in HMCAS group and $160( \pm 35)$ minutes in the nonHMCAS group $(\mathrm{p}=0.55)$. Pre-stroke $\mathrm{mRS}$ was 0 in both groups $(p=0.54)$. Patients in the HMCAS group had a trend towards a higher mean $( \pm \mathrm{SD})$ pre-treatment NIHSS score compared to

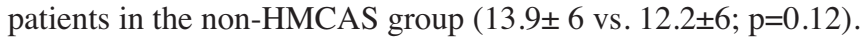
Accordingly, there were significantly more patients with severe strokes (NIHSS >10) in the HMCAS group compared to the nonHMCAS group $(48 / 64=75 \%$ vs. $35 / 66=53 \% ; p=0.009)$. At 24 hours post-rtPA treatment, the mean $( \pm \mathrm{SD})$ NIHSS score was

Table 2: The modified Rankin Score (mRS) dichotomized for good (mRS 0-2) and poor outcome (mRS 3-6) for patients with and without HMCAS

\begin{tabular}{lccc}
$\begin{array}{l}\text { 3-month outcome } \\
\text { Good outcome }\end{array}$ & $\begin{array}{c}\text { HMCAS(+) } \\
\mathbf{n = 6 4}\end{array}$ & $\begin{array}{c}\text { HMCAS(-) } \\
\mathbf{n = 6 6}\end{array}$ & $\begin{array}{c}\mathbf{P} \\
\text { value }\end{array}$ \\
$\begin{array}{l}\text { mRS 0-2 } \\
\text { Poor outcome } \\
\text { mRS 3-6 }\end{array}$ & 24 & 30 & 0.36 \\
\hline
\end{tabular}


$10.6( \pm 8)$ in the HMCAS group and $8.3( \pm 7)$ in non-HMCAS group $(\mathrm{p}=0.08)$. The length of hospital stay data was not normally distributed so a Mann-Whitney test was applied. Length of hospital stay was significantly greater in the HMCAS group compared with the non-HMCAS: $14( \pm 19)$ days vs. 9.73 $( \pm 22)$, respectively, $\mathrm{p}=0.012$.

There was no significant difference in the occurrence of any severe bleeding complication (systemic and intracranial combined) between the HMCAS group and non-HMCAS group ( $11 \%$ vs. $12 \%$; $=0.78)$. There was one fatal intracerebral hemorrhage (ICH) in the HMCAS group and two fatal ICH in the non-HMCAS group $(\mathrm{p}=0.57)$.

At the three-month follow-up, there was no significant difference in median mRS between patients in the HMCAS group and the non-HMCAS group (4 vs. 3; p=0.412), as well as in the percentage of patients with good outcome (i.e. mRS 0-2) ( $24 \%$ vs. $30 \%$; $=0.36$ ) (Figure 1 , Table 2 ). A multiple logistic regression analysis was done with the mRS dichotomized as good (0-2) and poor (3-6) outcome as the dependent variable. Age, gender, pre-treatment NIHSS, presence or absence of a HMCAS, as well as presence or absence of vascular risk factors were entered in the model as independent variables. The presence of a HMCAS was not an independent predictor of poor outcome. The only independent predictor of poor outcome (i.e. mRS 3-6) was pre-treatment NIHSS score $(\mathrm{p}<0.001)$.

\section{Discussion}

We observed that patients with a HMCAS receiving IV rtPA within three hours of symptom onset did not fare worse at three months than patients with no HMCAS, although more patients with a HMCAS had more severe strokes at baseline. A systematic review of early CT signs of stroke revealed that the presence of a hyperattenuated artery was associated with a worse outcome at six months ${ }^{28}$ but a number of studies have shown better outcomes when patients with HMCAS were treated with IV rtPA. ${ }^{12,13}$ and that a HMCAS was not an independent predictor of poor outcome after IV thrombolysis. ${ }^{12}$ In our study, a multivariable regression model revealed that pre-treatment NIHSS was the best predictor of outcome at three months. Schellinger showed that initial NIHSS and age were the best predictors of recanalization. ${ }^{29}$ In Albers' ${ }^{10}$ study, other variables in conjunction with HMCAS such as age greater than 85, high mean arterial blood pressure, and a greater than $33 \%$ of the MCA territory hypodensity were important for predicting poor outcome.

The efficacy of intravenous thrombolytic treatment within three-hours of symptom onset in patients with a HMCAS continues to be questioned. It is not known whether there is justification to withhold some of the IV rtPA treatment within three hours of symptom onset if a HMCAS is present and confirm the MCA occlusion with angiography to eventually precede with IA catheter-directed reperfusion strategies. This has been tested in a pilot randomized placebo-controlled trial during which IV rtPA or placebo was started within three hours of stroke onset followed by IA rtPA. ${ }^{19}$ The study demonstrated that IV+IA treatment is feasible and provides better recanalization compared to placebo or IA treatment. However, combined treatment was not associated with improved clinical outcomes and was associated with higher mortality. A recent retrospective study performed in a relatively small sample size as ours demonstrated that patients with a HMCAS did better at three months if they received IA rtPA treatment beyond three hours compared to patients showing a HMCAS who were treated with IV rtPA within three hours. ${ }^{20}$ The percentage of good outcome at three months, however, was higher in our HMCAS group compared to their HMCAS group treated with IV rtPA (37\% vs. 23\%), with similar mortality rates at three-month (22\% vs. $23 \%)$. Time-totreatment was also similar between our patients and their IV rtPA cohort (160 vs. 156 minutes). Our patients had a lower mean admission NIHSS score compared to their HMCAS group treated with IV rtPA (13.9 vs. 17.5), but our patients were older (mean age was 73 vs. 61), and this might explain the similar rate of bad outcomes we observed despite less severe strokes (pretreatment NIHSS score). Interestingly, the authors had a higher percentage of patients with cardioembolic strokes in the IA rtPAtreated group compared with the IV rtPA-treated one $(67 \%$ vs. $47 \%)$, despite a similar (20\%) occurrence of atrial fibrillation in both groups. In addition, there were more patients in the "large artery disease" group in the IV rtPA-treated group compared to IA rtPA-treated ones (19\% vs. $9 \%$ ). Schellinger et $\mathrm{al}^{29}$ have shown that having a cardioembolic stroke did significantly increase the odds of recanalization. Taken together, these observations suggest that IA rtPA treatment might be beneficial in a subset of patients severely impaired, not excessively old, whose stroke is attributable to cardioembolic disease. Prospective studies would better clarify this important issue.

Our study has several limitations. Due to the retrospective nature of the study, there may be selection bias due to missing patient information. Furthermore, our study lacked angiographic confirmation of vessel patency, therefore a proportion of patients in the non-HMCAS group may in fact have had a major vessel occlusion and we would have been unaware of this condition. This could affect outcomes in the non-HMCAS group, making them more similar to patients in the HMCAS group. Our cohort sample was relatively small as compared to previous studies. This was mainly due to lack of available pre-treatment CT scans and missing data at three-month follow-up. Since our institution is a tertiary referral centre, acute stroke patients who are candidates for thrombolysis may arrive with a CT scan performed in a peripheral hospital, which may be unavailable for subsequent review. Furthermore, there are many patients who come for thrombolysis but since they reside far from our institution, all clinical follow-up and further imaging is done closer to home. In addition, our hospital has only recently converted to a filmless picture archiving and communication system (PACS) system and some films may not have been archived properly and so have gone missing. Another limitation of this study is that we did not look at other early ischemic changes such as swelling of the affected hemisphere, loss of differentiation between grey and white matter, or effacement of cerebral sulci. However, the HMCAS alone is the most predictive of proximal MCA occlusion, and is the most obvious of the early CT signs of stroke, the others being sometimes subtle and missed even by experienced observers. ${ }^{2}$

In summary, until the results of a prospective ongoing study comparing IV vs. IA treatment within six hours of symptom onset are made available ${ }^{21}$, the presence of a HMCAS on pretreatment CT scan should not prevent clinicians from timely IV 
treatment with rtPA. These patients, who present with severe strokes, seem to have similar rates of good recovery as compared to patients not showing the clot-sign on pre-treatment CT scan.

\section{REFERENCES}

1. The National Institute of Neurological Disorders and Stroke rt-PA Stroke Study Group. Tissue plasminogen activator for acute ischemic stroke. N Engl J Med. 1995;333:1581-7.

2. Garg N, Eshkar N, Tanenbaum L, Cohen B, Sen S. Computed tomography angiographic correlates of early computed tomography signs in acute ischemic stroke. J Neuroimaging. 2004; $14: 242-5$

3. Tomsick T, Brott T, Barsan W, Broderick J, Haley EC, Spilker J, et al. Prognostic value of the hyperdense middle cerebral artery sign and stroke scale score before ultraearly thrombolytic therapy. AJNR Am J Neuroradiol. 1996;17:79-85.

4. Pressman BD, Tourje EJ, Thompson JR. An early CT sign of ischemic infarction: increased density in a cerebral artery. AJR Am J Roentgenol. 1987; 149:583-6.

5. Schuierer G, Huk W. The unilateral hyperdense middle cerebral artery: an early CT-sign of embolism or thrombosis. Neuroradiology. 1988;30:120-2.

6. Tomsick TA, Brott TG, Olinger CP, Barsan W, Spilker J, Eberle R, et al. Hyperdense middle cerebral artery: incidence and quantitative significance. Neuroradiology. 1989;31:312-5.

7. Tomsick T, Brott T, Barsan W, Broderick J, Haley EC, Spilker J. Thrombus localization with emergency cerebral CT. AJNR Am J Neuroradiol. 1992;13:257-63.

8. Schuknecht B, Ratzka M, Hofmann E. The "dense artery sign"-major cerebral artery thromboembolism demonstrated by computed tomography. Neuroradiology. 1990;32:98-103.

9. Bastianello S, Pierallini A, Colonnese C, Brughitta G, Angeloni U, Antonelli M, et al. Hyperdense middle cerebral artery CT sign. Comparison with angiography in the acute phase of ischemic supratentorial infarction. Neuroradiology. 1991;33:207-11.

10. Albers GW, Bates VE, Clark WM, Bell R, Verro P, Hamilton SA. Intravenous tissue-type plasminogen activator for treatment of acute stroke: the Standard Treatment with Alteplase to Reverse Stroke (STARS) study. JAMA. 2000;283:1145-50.

11. Derex L, Hermier M, Adeleine P, Pialat JB, Wiart M, Berthezene Y, et al. Clinical and imaging predictors of intracerebral haemorrhage in stroke patients treated with intravenous tissue plasminogen activator. J Neurol Neurosurg Psychiatry. 2005;76:70-5.

12. Manelfe C, Larrue V, von Kummer R, Bozzao L, Ringleb P, Bastianello S, et al. Association of hyperdense middle cerebral artery sign with clinical outcome in patients treated with tissue plasminogen activator. Stroke. 1999;30:769-72.

13. Qureshi AI, Ezzeddine MA, Nasar A, Suri MF, Kirmani JF, Janjua $\mathrm{N}$, et al. Is IV tissue plasminogen activator beneficial in patients with hyperdense artery sign? Neurology. 2006;66:1171-4.

14. Berge E, Nakstad PH, Sandset PM. Large middle cerebral artery infarctions and the hyperdense middle cerebral artery sign in patients with atrial fibrillation. Acta Radiol. 2001;42:261-8.

15. Derex L, Nighoghossian N, Hermier M, Adeleine P, Froment JC, Trouillas P. Early detection of cerebral arterial occlusion on magnetic resonance angiography: predictive value of the baseline NIHSS score and impact on neurological outcome. Cerebrovasc Dis. 2002;13:225-9.
16. Wolpert SM, Bruckmann H, Greenlee R, Wechsler L, Pessin MS, del Zoppo GJ. Neuroradiologic evaluation of patients with acute stroke treated with recombinant tissue plasminogen activator. The rt-PA Acute Stroke Study Group. AJNR Am J Neuroradiol. 1993;14:3-13.

17. Roberts HC, Dillon WP, Furlan AJ, Wechsler LR, Rowley HA, Fischbein NJ, et al. Computed tomographic findings in patients undergoing intra-arterial thrombolysis for acute ischemic stroke due to middle cerebral artery occlusion: results from the PROACT II trial. Stroke. 2002;33:1557-65.

18. Lewandowski CA, Frankel M, Tomsick TA, Broderick J, Frey J, Clark W, et al. Combined intravenous and intra-arterial r-TPA versus intra-arterial therapy of acute ischemic stroke: Emergency Management of Stroke (EMS) Bridging Trial. Stroke. 1999;30:2598-605.

19. Zaidat OO, Suarez JI, Santillan C, Sunshine JL, Tarr RW, Paras VH, et al. Response to intra-arterial and combined intravenous and intra-arterial thrombolytic therapy in patients with distal internal carotid artery occlusion. Stroke. 2002;33:1821-6.

20. Mattle HP, Arnold M, Georgiadis D, Baumann C, Nedeltchev K, Benninger $\mathrm{D}$, et al. Comparison of intraarterial and intravenous thrombolysis for ischemic stroke with hyperdense middle cerebral artery sign. Stroke. 2008;39:379-83.

21. Ciccone A, Boccardi E, Cantisani TA, Coppola C, Gatti A, Guccione A, et al. A randomized comparison of intra-arterial with intravenous thrombolysis for acute ischemic dtroke: The SYNTHESIS trial. International Stroke Conference. New Orleans: 2005.

22. Saposnik G, Young B, Silver B, Di Legge S, Webster F, Beletsky V, et al. Lack of improvement in patients with acute stroke after treatment with thrombolytic therapy: predictors and association with outcome. JAMA. 2004;292:1839-44.

23. Merino JG, Silver B, Wong E, Foell B, Demaerschalk B, Tamayo A, et al. Extending tissue plasminogen activator use to community and rural stroke patients. Stroke. 2002;33:141-6.

24. Foell RB, Silver B, Merino JG, Wong EH, Demaerschalk BM, Poncha $\mathrm{F}$, et al. Effects of thrombolysis for acute stroke in patients with pre-existing disability. CMAJ. 2003; 169:193-7.

25. Brott T, Adams HP, Jr., Olinger CP, Marler JR, Barsan WG, Biller $\mathrm{J}$, et al. Measurements of acute cerebral infarction: a clinical examination scale. Stroke. 1989; 20:864-70.

26. Adams HP, Jr, Davis PH, Leira EC, Chang KC, Bendixen BH, Clarke WR, et al. Baseline NIH Stroke Scale score strongly predicts outcome after stroke: A report of the Trial of Org 10172 in Acute Stroke Treatment (TOAST). Neurology. 1999;53: 126-31.

27. Bonita R, Beaglehole R. Recovery of motor function after stroke. Stroke. 1988;19:1497-500.

28. Wardlaw JM, Mielke O. Early signs of brain infarction at CT: observer reliability and outcome after thrombolytic treatment-systematic review. Radiology. 2005;235:444-53.

29. Schellinger PD, Chalela JA, Kang DW, Latour LL, Warach S. Diagnostic and prognostic value of early MR imaging vessel signs in hyperacute stroke patients imaged $<3$ hours and treated with recombinant tissue plasminogen activator. AJNR Am J Neuroradiol. 2005;26:618-24. 